\title{
Ligand-to-Copper Charge Transfer: A General Catalytic Approach to
}

\section{Aromatic Decarboxylative Functionalization}

Tiffany Q. Chen, ${ }^{1}$ P. Scott Pedersen, ${ }^{1}$ Nathan W. Dow, ${ }^{1}$ Remi Fayad,,${ }^{2}$ Cory E. Hauke, ${ }^{2}$ Michael

C. Rosko, ${ }^{2}$ Evgeny O. Danilov, ${ }^{2}$ David C. Blakemore, ${ }^{3}$ Anne-Marie Dechert-Schmitt,${ }^{3}$ Thomas

Knauber, ${ }^{3}$ Felix N. Castellano, ${ }^{2}$ David W. C. MacMillan ${ }^{1 *}$

${ }^{1}$ Merck Center for Catalysis at Princeton University, Princeton, New Jersey 08544, USA.

${ }^{2}$ Department of Chemistry, North Carolina State University, Raleigh, North Carolina 27695, USA.

${ }^{3}$ Worldwide Research and Development, Pfizer Inc., Eastern Point Road, Groton, Connecticut 06340, USA.

ABSTRACT: Aryl carboxylic acids are valuable, stable, and abundant functional handles in organic synthesis. Historically, their activation with established two-electron methods requires forcing conditions, and such protocols are limited in scope. In contrast, we envisioned that copper's ability to generate open-shell species through ligand-to-metal charge transfer (LMCT), combined with its unique capacity to act as a potential aroyloxy and aryl radical reservoir, could mediate facile light- and copper-enabled aromatic decarboxylative functionalization by mitigating undesired reactivity of radical intermediates formed during aromatic decarboxylation. We report herein a general copper-LMCT open-shell activation platform for aromatic halodecarboxylation. Catalytic decarboxylative chlorination, bromination, and iodination of diverse (hetero)aryl carboxylic acids have been achieved to provide broadly used electrophilic cross-coupling handles from widely available aromatic acid precursors. Notably, decarboxylative fluorination of aryl carboxylic acids - a long-standing challenge in the field of organic synthesis - is readily accessible over a wide breadth of (hetero)aryl substrates. Ultrafast transient absorption (TA) spectroscopy experiments in combination with steady-state UV-vis spectroscopy studies are consistent with the proposed copper-LMCT mechanism, supporting the mechanistic basis of this activation platform. 
The functionalization of carboxylic acids has emerged as a vibrant field of research in organic synthesis over the past decade. Their bench stability, diversity, and ubiquity in nature and in pharmaceuticals and functional materials render them privileged and attractive building blocks. In particular, redox chemistry platforms such as metallaphotoredox catalysis have enabled general strategies for the functionalization of aliphatic carboxylic acids, which encompass diverse transformations including alkylation, ${ }^{1}$ arylation, ${ }^{2-4}$ amination, ${ }^{5}$ and trifluoromethylation. ${ }^{6}$ In contrast, the development of a general platform for the direct activation of benzoic acids remains a long-standing challenge..$^{7-8}$ While the decarboxylative functionalization of aliphatic carboxylic acids is readily achievable through photoredox catalysis, several limitations render photocatalytic platforms ineffective in the activation of their aromatic counterparts. Due to the more positive reduction potential of aryl carboxylates (e.g., $E_{\mathrm{p} / 2}\left[\mathrm{PhCO}_{2}-/ \mathrm{PhCO}_{2}{ }^{-}\right]=+1.46 \mathrm{~V}$ vs SCE in $\left.\mathrm{CH}_{3} \mathrm{CN}\right),{ }^{9}$ aroyloxy radicals are susceptible to rapid back-electron transfer (BET) with a photoredox catalyst, regenerating the starting carboxylate and the ground-state photoredox catalyst. ${ }^{10}$ Additionally, $O$ -

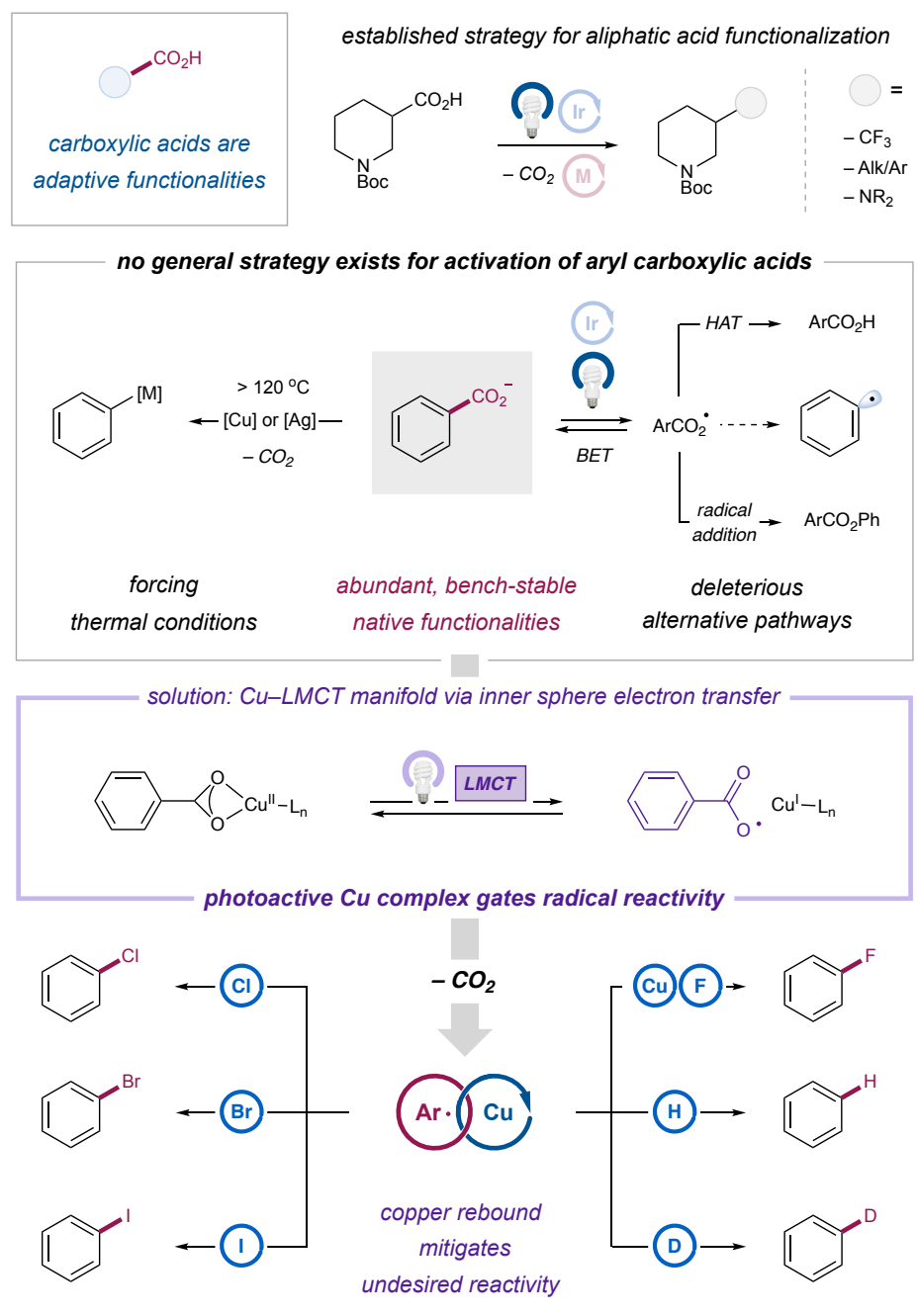

this work: a general platform for decarboxylative functionalization of aryl carboxylic acids

Figure 1. A general platform for decarboxylative functionalization of aryl carboxylic acids via copperligand-to-metal charge transfer (LMCT). 
centered aroyloxy radical intermediates readily undergo hydrogen atom transfer $(\mathrm{HAT})\left(k \approx 10^{7}\right.$ $\mathrm{M}^{-1} \mathrm{~s}^{-1}$ ) to reform the parent aryl carboxylic acid, precluding successful decarboxylation which occurs at comparatively sluggish rates $\left(k \approx 10^{4}\right.$ to $\left.10^{6} \mathrm{M}^{-1} \mathrm{~s}^{-1}\right) \cdot{ }^{11-12}$ Furthermore, while thermal copper- ${ }^{13-16}$ or silver- ${ }^{17,18}$ mediated decarboxylation methods have been widely explored for aryl carboxylic acids, these methods exhibit poor generality (often limited to ortho-substituted or electron-deficient aryl carboxylic acids) and require forcing conditions (e.g., heating to above 120 ${ }^{\circ} \mathrm{C}$ and/or using highly oxidizing stoichiometric reagents such as persulfates). ${ }^{14-15,19-20}$ As aryl carboxylic acids are valuable motifs for late-stage functionalization- due to their commercial availability and bench stability, as well as their accessibility from "masked" precursors such as tolyl and aryl ester moieties ${ }^{7}$ - the development of a general platform for their activation would enable access to this highly desirable yet historically elusive class of substrates and highlight their utility as synthetically powerful adaptive functionalities.

Ligand-to-metal charge transfer (LMCT) processes have been employed to generate reactive open-shell intermediates from coordination complexes upon light irradiation. ${ }^{21-27}$ Notably, aliphatic copper(II)-carboxylate complexes can undergo LMCT upon irradiation through innersphere electron transfer from a carboxylate ligand to copper(II), forming a dissociated carboxyl radical and copper(I). We envisioned that this photochemical process could serve as a key design principle in the development of a general light- and copper-enabled decarboxylative functionalization platform for aromatic carboxylic acids. We were intrigued by the possibility of combining the unique photochemistry and reactivity of copper to mitigate undesired reactivity of radical intermediates formed in the process of aromatic decarboxylation. Specifically, we expected that not only could a copper-carboxylate complex generate aryl radical intermediates upon LMCT, but the inherent radical-trapping capacity of copper could facilitate a protective, reversible radical 
rebound pathway that curtails detrimental aroyloxy radical HAT. Similarly, upon decarboxylation, a reversible radical rebound pathway could gate and minimize undesired reactivity of the resulting aryl radical. Previous studies of thermal $\mathrm{Cu}$-mediated aromatic decarboxylation have isolated distinct copper-aryl intermediates, supporting the possibility of leveraging these intermediates as a design element. ${ }^{20}$ In addition, copper(II)-alkyl complexes have been demonstrated to undergo reversible homolysis to form an alkyl radical and copper(I) ${ }^{28-29}$ Copper's unique ability to generate open-shell species through LMCT, combined with its facility to act as a potential aroyloxy and aryl radical reservoir, could thus serve as a powerful and general platform for light- and coppermediated decarboxylative activation of aromatic carboxylic acids.

We anticipated that aryl radicals formed from aromatic decarboxylation could be subsequently functionalized via radical trapping reagents or radical rebound with copper to furnish a versatile copper-aryl intermediate for diverse functionalization. Accessing a suite of aryl halide products directly from aryl carboxylic acids would be an important synthetic advance, as no current aromatic halodecarboxylation strategies can broadly engage both electron-rich and electron-poor (hetero)aryl acids under mild reaction conditions. ${ }^{7}$ Furthermore, we envisioned that given the tremendous importance of site-selective aromatic fluorination in drug discovery, ${ }^{30-31}$ expanding the generality of such a halodecarboxylation protocol to aromatic fluorodecarboxylation would be noteworthy. ${ }^{32}$ Altogether, the development of a general activation platform for aryl carboxylic acids would demonstrate the importance of these building blocks as powerful adaptable synthons, capable of delivering versatile coupling handles and valuable pharmacophores alike.

With this copper-LMCT strategy in mind, we expected that a catalytic manifold for aromatic halodecarboxylation could be achieved by utilizing a copper(I) salt, stoichiometric oxidant, and an electrophilic halogenating reagent able to undergo halogen atom transfer to an aryl radical. We 
began by investigating the feasibility of catalytic bromodecarboxylation. Following a survey of various copper salts, oxidants, solvents, and bromination reagents, $365 \mathrm{~nm}$ LED irradiation of 4(trifluoromethyl)benzoic acid, $\left[\mathrm{Cu}(\mathrm{MeCN})_{4}\right] \mathrm{BF}_{4}(20 \mathrm{~mol} \%)$, 1-fluoro-1,3,5-trimethylpyridinium tetrafluoroborate (NFTPT) (1 equiv.), and 1,3-dibromo-5,5-dimethylhydantoin (DBDMH) (0.75 equiv.) in $\mathrm{CH}_{3} \mathrm{CN}$ delivered the desired bromodecarboxylated product $\mathbf{1}$ in 85\% yield (Figure 1). Key to attaining good reactivity was the use of a copper(I) or copper(II) salt (in particular, $\left[\mathrm{Cu}(\mathrm{MeCN})_{4}\right] \mathrm{BF}_{4}$, although a variety of copper salts provided good efficiencies), a soluble singleelectron oxidant (such as an $N$-fluorinated oxidant or dicumyl peroxide), an electrophilic bromination reagent, and $\mathrm{CH}_{3} \mathrm{CN}$ as solvent to minimize HAT by reactive carboxylate-derived radical species and putatively stabilize the active copper complex. The $\left[\mathrm{Cu}(\mathrm{MeCN})_{4}\right] \mathrm{BF}_{4}$ loading could be decreased to as low as $5 \mathrm{~mol} \%$ and still furnish moderate yields (40\%), and no conversion was observed in control reactions without copper or without light irradiation.

With these observations and optimized conditions in hand, we next evaluated the scope of this bromodecarboxylation reaction. We were gratified to find that both electron-rich and electrondeficient substrates were readily functionalized $(\mathbf{1 - 5}, 68$ to $80 \%$ yield), a limitation for many existing aryl decarboxylation strategies. ${ }^{7}$ With substrates bearing electron-donating substituents, using bromotrichloromethane $\left(\mathrm{CCl}_{3} \mathrm{Br}\right)$ instead of an $\mathrm{N}$-brominated reagent suppresses deleterious electrophilic aromatic substitution that leads to overbromination of the product. Moreover, not only were sterically encumbered benzoic acids, including ortho- and ortho,ortho-disubstituted acids (6 and 7, 79\% and 53\% yield, respectively) competent substrates but also meta- and parasubstituted acids, which are typically poor substrates in thermal decarboxylation methods due to a lack of ground-state destabilization. ${ }^{33}$ With respect to the scope of heterocyclic acids, all 


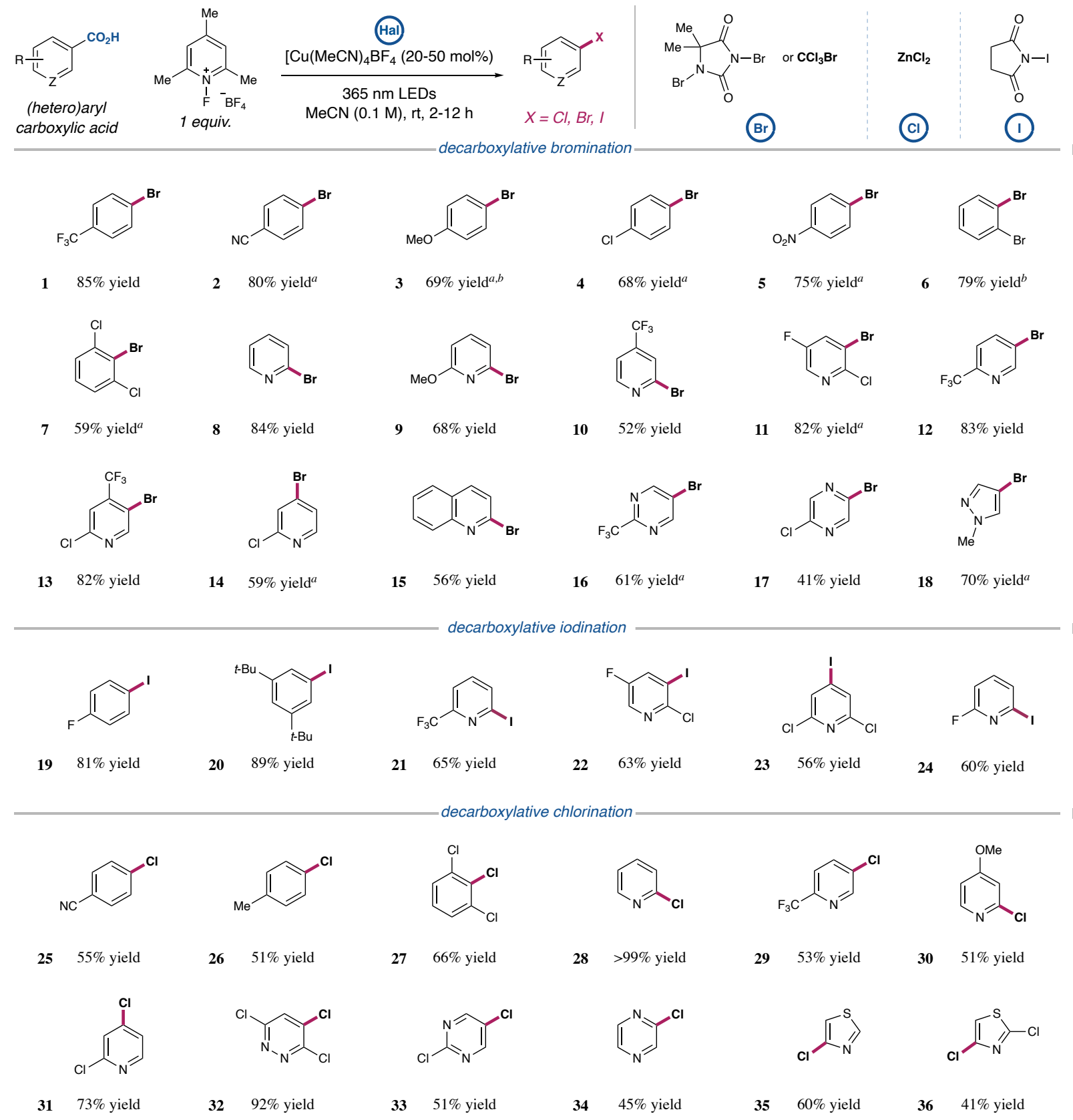

Figure 2. Bromo-, chloro-, and iododecarboxylation substrate scope. All yields determined by ${ }^{1} \mathrm{H}-$ NMR of crude reaction mixture with respect to an internal standard, unless otherwise noted.

asolated yield. ${ }^{\text {b }} \mathrm{With} \mathrm{CCl}_{3} \mathrm{Br}$ as bromination reagent (3 equiv.).

regioisomers of pyridine carboxylic acids, including picolinic acids (8 - 10, 84-52\% yield), nicotinic acids $(\mathbf{1 1}-\mathbf{1 3}, 82-83 \%$ yield $)$, and isonicotinic acids (14, 76\% yield), exhibited excellent reactivity. More complex heterocyclic acids (16 and 17, 63\% and 41\% yield, respectively) furnished the corresponding brominated product with high efficiency, as did 5-membered 
heterocycles such as pyrazole $(\mathbf{1 8}, 70 \%$ yield). Additionally, we successfully translated this protocol to iododecarboxylation by employing $N$-iodosuccinimide as the electrophilic halogenation reagent. We were pleased to find that iododecarboxylated products of benzoic acids (19 and $\mathbf{2 0}, 81 \%$ and $89 \%$ yield, respectively) and every pyridine acid regioisomer (21 - $\mathbf{2 4}, 56-$ $65 \%$ yield) could be obtained in efficient yields.

In contrast, $N$-chlorosuccinimide and other electrophilic chlorination reagents were less effective for chlorodecarboxylation, which can be attributed to the higher bond dissociation energy (BDE) of the $\mathrm{N}-\mathrm{Cl}$ bond relative to $\mathrm{N}-\mathrm{Br}$ or $\mathrm{N}-\mathrm{I}$ and a higher barrier to atom transfer. However, we found that we could efficiently access chlorodecarboxylation products by utilizing stoichiometric $\mathrm{ZnCl}_{2}$. Under these conditions, we hypothesize that chlorination proceeds through ligand exchange of chloride on copper from $\mathrm{ZnCl}_{2}$, then subsequent $\mathrm{C}-\mathrm{Cl}$ reductive elimination from a high-valent copper intermediate or outer-sphere chlorine atom transfer, introducing an alternative potential pathway for copper-catalyzed functionalization within this manifold. On investigating the scope of this transformation, chlorodecarboxylation could be readily achieved with electronically diverse benzoic acids ( $\mathbf{2 5}-\mathbf{2 7}, 51-66 \%$ yield); 2-, 3-, and 4-substituted pyridine acids (28 - 31, 51-99\% yield); dinitrogen-containing heterocyclic acids such as pyridazine (32, 92\% yield), pyrimidine (33, 51\% yield) and pyrazine acids (34, 45\% yield); and 5-membered scaffolds such as thiazole4-carboxylic acids (35 and 36, 60\% and 41\% yield, respectively). In combination, these halodecarboxylation protocols enable the rapid diversification of aryl carboxylic acids into a library of valuable and diverse haloarene coupling partners using a unified copper-LMCT platform.

Based on conditions required for the chlorodecarboxylation protocol, we recognized the potential for copper to serve multiple roles in this platform: to accomplish LMCT activation and 
subsequent aryl radical capture and cross-coupling. ${ }^{32}$ As reductive elimination from high-valent copper complexes is facile for a range of coupling partners, ${ }^{34}$ and copper-mediated radical capture/reductive elimination sequences have been demonstrated to be successful for challenging couplings, such as $\mathrm{C}-\mathrm{CF}_{3}$ bond formation, ${ }^{35}$ we envisioned that at higher loadings, copper could facilitate sequential aryl radical formation, radical capture, and $\mathrm{C}\left(\mathrm{sp}^{2}\right)-\mathrm{F}$ cross-coupling to enable fluorodecarboxylation. Indeed, increasing the copper loading to 3 equivalents was successful in delivering a previously elusive platform for aromatic fluorodecarboxylation, with the standard pyridinium oxidant serving as a bench-stable, in situ source of fluoride. In some instances, the addition of cesium fluoride enhanced the efficiency of decarboxylative fluorination by reducing aryl ester formation, a byproduct generated via sequential decarboxylation and $\mathrm{C}-\mathrm{O}$ bond formation. This approach proved competent for the fluorination of electron-rich and electrondeficient benzoic acids with different substitution patterns (37-42, 52-74\% yield). Most notably, in line with trends elucidated for the previous halogenation reactions, this copper-mediated protocol was effective across diverse classes of heterocyclic substrates, expediently furnishing fluorinated derivatives of pyridine (43 - 46, 48-70\% yield), pyrimidine (47 and $48,66 \%$ and $40 \%$ yield, respectively), pyrazine (49-51, 69-76\% yield) pyridazine (52, 52\% yield), bicyclic quinoxaline $(\mathbf{5 3}, \mathbf{6 6 \%}$ yield) and isoquinoline scaffolds $(\mathbf{5 4}, 57 \%$ yield). We believe this fluorination platform, which can be readily performed on the bench top under ambient conditions, represents an important synthetic advance as one of the most broadly applicable set of aromatic fluorodecarboxylation conditions currently reported for heteroaryl acids.

Finally, we demonstrate the unique modularity of aryl carboxylic acids as synthons in a programmable synthetic sequence and the generality of this mild copper-catalyzed LMCT decarboxylative functionalization protocol. Tolyl moieties in complex molecules such as 


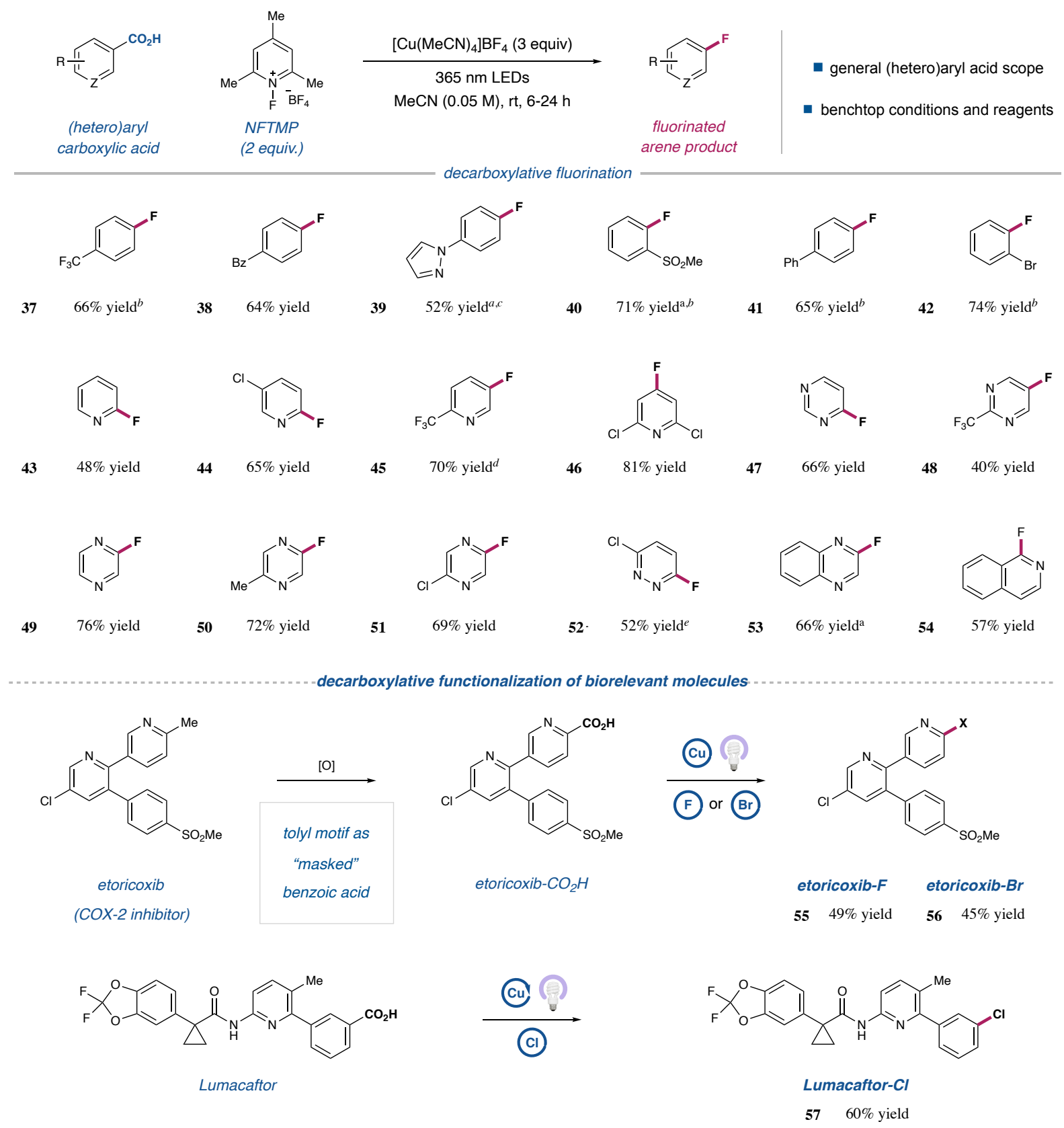

Figure 3. Fluorodecarboxylation substrate scope. All yields determined by ${ }^{19} \mathrm{~F}-\mathrm{NMR}$ of crude reaction mixture with respect to an internal standard, unless otherwise noted. asolated yield. ${ }^{b}$ With one equivalent of CsF. ${ }^{~}$ Run at $0.038 \mathrm{M}$. ${ }^{d}$ Run with 2.5 equiv. NFTPT. ${ }^{e}$ Run at $0.063 \mathrm{M}$.

etoricoxib can be viewed as "masked" benzoic acids and revealed upon mild oxidation. Etoricoxib$\mathrm{CO}_{2} \mathrm{H}$ was readily obtained upon oxidation with $\mathrm{KMnO}_{4}$, and subjecting this substrate to bromodecarboxylation and fluorodecarboxylation conditions yielded the fluorinated and brominated derivatives in useful efficiencies ( $\mathbf{5 5}$ and $\mathbf{5 6}, 49 \%$ and $45 \%$ yield, respectively). 
Similarly, subjecting the drug Lumacaftor to chlorodecarboxylation conditions gave the respective chlorinated product in good yield (57 60\% yield). These examples highlight the general nature of this copper-catalyzed LMCT strategy, which enables (hetero)aryl acid decarboxylative functionalization with broad tolerance for functional groups and structural complexity to afford a valuable library of halogenated complex molecules.

A proposed mechanism for the catalytic decarboxylative functionalization manifold is described in Figure 4A. Upon combination of a copper(I) catalyst with an oxidant and the aryl carboxylic acid substrate, aryl carboxylate-copper(II) complex $\mathbf{5 8}$ could be formed. Photoexcitation of this complex followed by LMCT would induce homolysis of the copper-oxygen bond, generating reduced $\mathrm{Cu}(\mathrm{I})$ and an aroyloxy radical in a solvent cage (59). While BET would regenerate the ground state of the original aryl-carboxylate-Cu(II) complex, decarboxylation of the aroyloxy radical could occur in the solvent cage to form an aryl radical and $\mathrm{CO}_{2}$. The aryl radical could then either reversibly rebound onto copper or undergo cage escape to react with a halogenation reagent via atom transfer to furnish the desired halogenated arene. Re-ligation with aryl carboxylate and single-electron oxidation of $\mathrm{Cu}(\mathrm{I})(\mathbf{6 0})$ would close the copper catalytic cycle. Under conditions using catalytic copper for chlorodecarboxylation or conditions using superstoichiometric copper for fluorodecarboxylation, aryl radical capture by a putative copper(II)-halide complex could generate high-valent copper(III), and subsequent $\mathrm{C}-\mathrm{X}$ reductive elimination would deliver the desired aryl halide.

We next sought to elucidate unique features of this mechanism, including intermediacy of an aryl radical (Figure 4B). Catalytic hydrodecarboxylation of 4-(tert-butyl)benzoic acid could be achieved using $\mathrm{Cu}(\mathrm{OTf})_{2}(10 \mathrm{~mol} \%)$ and stoichiometric $\mathrm{NFSI}$ in $\mathrm{CH}_{3} \mathrm{CN}(\mathbf{6 1}, 76 \%$ yield), providing a facile strategy for the reduction of traceless carboxylate directing groups in arenes. ${ }^{11,36}$ 
Additionally, deuterodecarboxylation - with quantitative deuterium incorporation - was attained when the reaction was conducted in $\mathrm{CD}_{3} \mathrm{CN}$, consistent with $\mathrm{HAT}$ from solvent in the hydrodecarboxylation reaction $(\mathbf{6 2}, 20 \%$ yield). Similarly, we observed the formation of the Giese reaction product $\mathbf{6 3}$ on addition of diethyl ethylidenemalonate (3 equiv.) to the reaction mixture. Finally, we demonstrated that near-quantitative yield of the aryl ester product $\mathbf{6 4}$ - resulting from aryl carboxylic acid decarboxylative homocoupling - could be obtained under conditions similar to those used in the fluorination reaction by simply replacing NFTPT with a non-fluorinated oxidant, such as dicumyl peroxide. This latter result is consistent with $\mathrm{C}-\mathrm{O}$ reductive elimination from copper, lending support to operative reductive elimination pathways under this manifold.

To investigate the key LMCT event, static UV-vis and transient absorption (TA) spectroscopy experiments were conducted (Figure 4C). In static UV-vis spectra, $\mathrm{Cu}(\mathrm{II})$ coordination complexes possess a diagnostic absorption band between $600 \mathrm{~nm}$ to $1000 \mathrm{~nm}$ assigned to ligand-field (d-d) transitions, a feature that is not observed in $\mathrm{Cu}(\mathrm{I}) \mathrm{d}^{10}$ species. Monitoring the steady-state absorption of the reaction mixture irradiated with $370 \mathrm{~nm}$ light would thus elucidate the oxidation state of copper. On addition of NFSI to $\left[\mathrm{Cu}(\mathrm{MeCN})_{4}\right] \mathrm{BF}_{4}$ and prior to illumination $(\mathrm{t}=0)$, a strong absorption band at $700 \mathrm{~nm}$ was observed, indicating the immediate formation of $\mathrm{Cu}(\mathrm{II})$ via oxidation of $\mathrm{Cu}(\mathrm{I})$. During two hours of continuous irradiation, this absorption band gradually redshifted to $800 \mathrm{~nm}$ and eventually decayed, indicating photoreduction of $\mathrm{Cu}(\mathrm{II})$ to $\mathrm{Cu}(\mathrm{I})$ via $\mathrm{LMCT}$ over the course of the reaction. In the absence of irradiation, no change in the absorption spectrum was observed over the two-hour experimental time window, demonstrating that light is needed for photoreduction of $\mathrm{Cu}(\mathrm{II})$.

Additionally, we leveraged TA spectroscopy to probe the intermediacy of the proposed photogenerated aroyloxy radical (Figure 4C). Benzoyloxy radicals, readily formed from their 
A

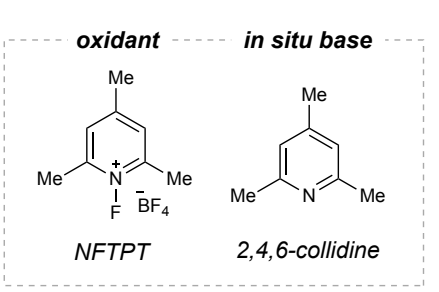

- catalytic halodecarboxylation

- broad substrate generality

- in situ base formation from oxidant
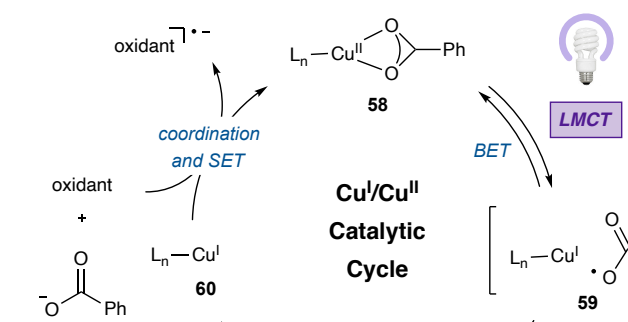

Catalytic

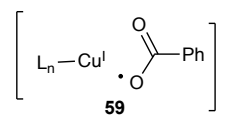

general platform for

activation of

aryl carboxylates via

copper(II)-LMCT

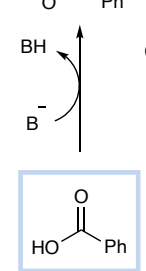

$1-\mathrm{CO}^{2}$
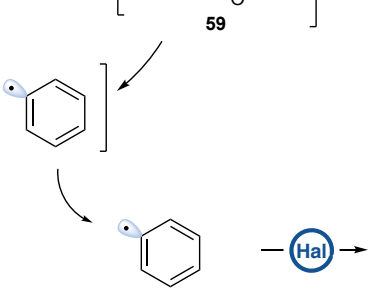

via halogen atom transfer

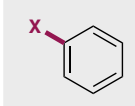

$X=C l, B r, I$ hydrodecarboxylation

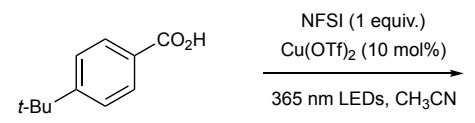

- strategy for aryl carboxylate reduction

C-C bond formation via Giese<smiles>O=C(O)c1ccc(F)cc1</smiles>

$\underset{\begin{array}{c}\text { diethyl ethylidenemalonate } \\ {\left[\mathrm{Cu}(\mathrm{MeCN})_{4}\right] \mathrm{BF}_{4}(20 \mathrm{~mol} \%)}\end{array}}{365 \mathrm{~nm} \mathrm{LEDs}, \mathrm{NFTPT}, \mathrm{CH}_{3} \mathrm{CN}}$

- consistent with aryl radical intermediacy

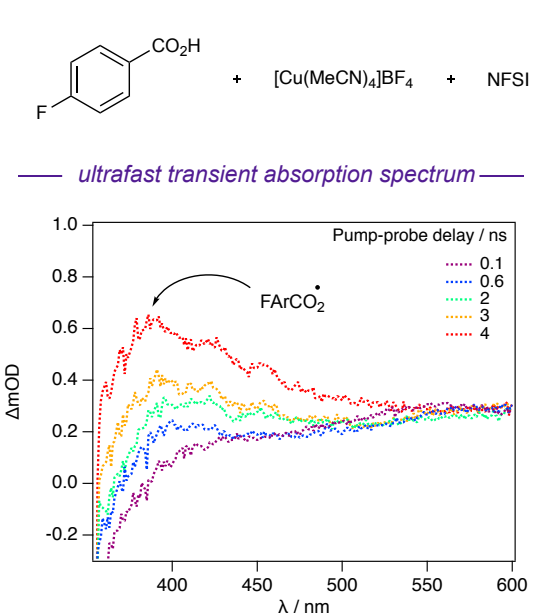

mechanistic features

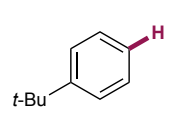

$\mathbf{6 1}, 77 \%$ yield

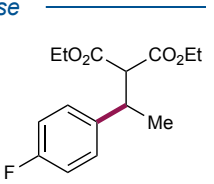

63, $23 \%$ yield

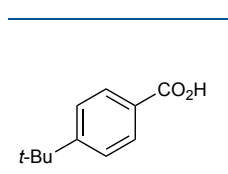

- $>99 \%$ deuterium incorporation

C-O bond formation

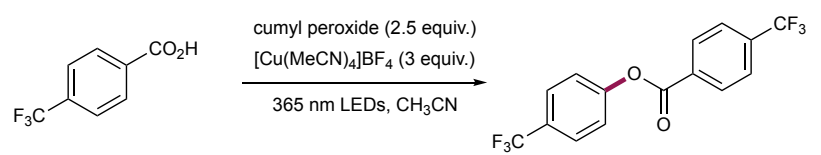

- cumyl peroxide promotes $\mathrm{C}-\mathrm{O}$ reductive elimination $\mathbf{6 4}, 98 \%$ yield

spectroscopic support for CU(II)-LMCT activation

Figure 4. A) Proposed mechanism for light-induced copper-catalyzed aromatic halodecarboxylation via copper-mediated ligand-to-metal charge transfer. B) Mechanistic features of this manifold. C) Spectroscopic support for $\mathrm{Cu}$ (II)-LMCT activation: ultrafast transient absorption spectrum and static UV-vis absorption spectrum. 
respective peroxides, feature a broad absorption band in the visible region between 500 to $800 \mathrm{~nm}$, a sharper absorption component with a maximum near $400 \mathrm{~nm}$, and a strong UV absorption centered at $320 \mathrm{~nm}$. The flowing solution generates a difference spectrum that closely resembles the benzoyloxy radical spectrum previously reported in the literature ${ }^{12}$ and which appeared in the prompt spectrum in this experiment, consistent with benzoyloxy radical formation within the laser pulse. The benzoyloxy radical was monitored using ultrafast TA spectroscopy $\left(\lambda_{\mathrm{ex}}=350 \mathrm{~nm}, 1.9\right.$ $\mathrm{mJ} / \mathrm{pulse}, 100 \mathrm{fs}$ fwhm) and formed over the $4 \mathrm{~ns}$ time delay window of the experiment. Notably, these features are not observed in the absence of the aryl carboxylic acid. In combination, these spectroscopic studies are consistent with aryl carboxylate-copper bond homolysis resulting from LMCT, supporting the mechanistic basis of this platform as a general manifold for activating and functionalizing previously elusive aryl carboxylic acid building blocks.

\section{AUTHOR INFORMATION}

\section{Corresponding Author}

*Prof. David W. C. MacMillan, dmacmill@princeton.edu, orcid.org/0000-0001-6447-0587

\section{ACKNOWLEDGEMENTS}

The authors are grateful for financial support provided by the National Institute of General Medical Sciences (NIGMS), the NIH (under Award R35GM134897-01), the Princeton Catalysis Initiative, BioLec, an Energy Frontier Research Center (U.S. Department of Energy, Office of Science, Office of Basic Energy Sciences, under Award DE-SC0019370), and kind gifts from Pfizer, Merck, Janssen, Bristol-Myers Squibb, Genentech, and Celgene. T. Q. C. thanks Bristol-Myers Squibb for a graduate fellowship. P. S. P. thanks the NSF for a predoctoral fellowship (Award DGE-1656466). The content is solely the responsibility of the authors and does not necessarily represent the official views of NIGMS. This work was performed in part at the Laboratory for Imaging and Kinetic Spectroscopy at North Carolina State University. 


\section{REFERENCES}

(1) Bloom, S.; Liu, C.; Kölmel, D. K.; Qiao, J. X.; Zhang, Y.; Poss, M. A.; Ewing, W. R.; MacMillan, D. W. C. Nat. Chem. 2018, 10 (2), 205-211.

(2) Chu, L.; Lipshultz, J. M.; MacMillan, D. W. C. Angew. Chem. Int .Ed. 2015, 54 (27), 79297933.

(3) Zuo, Z.; MacMillan, D. W. C. J. Am. Chem. Soc. 2014, 136 (14), 5257-5260.

(4) Zuo, Z.; Cong, H.; Li, W.; Choi, J.; Fu, G. C.; MacMillan, D. W. C. J. Am. Chem. Soc. 2016, $138(6), 1832-1835$.

(5) Liang, Y.; Zhang, X.; MacMillan, D. W. C. Nature 2018, 559 (7712), 83-88.

(6) Kautzky, J. A.; Wang, T.; Evans, R. W.; MacMillan, D. W. C. J. Am. Chem. Soc. 2018, $140(21), 6522-6526$.

(7) Varenikov, A.; Shapiro, E.; Gandelman, M. Chem. Rev. 2021, 121 (1), 412-484.

(8) Hu, X.-Q.; Liu, Z.-K.; Hou, Y.-X.; Gao, Y. iScience 2020, 23 (7), 101266.

(9) Li, H.; Subbotina, E.; Bunrit, A.; Wang, F.; Samec, J. S. M. Chem. Sci. 2019, 10 (12), 3681-3686.

(10) Kubosaki, S.; Takeuchi, H.; Iwata, Y.; Tanaka, Y.; Osaka, K.; Yamawaki, M.; Morita, T.; Yoshimi, Y. J. Org. Chem. 2020, 85 (8), 5362-5369.

(11) Chateauneuf, J.; Lusztyk, J.; Ingold, K. U. J. Am. Chem. Soc. 1988, 110 (9), 2877-2885.

(12) Chateauneuf, J.; Lusztyk, J.; Ingold, K. U. J. Am. Chem. Soc. 1988, 110 (9), 2886-2893.

(13) Shepard, A. F.; Winslow, N. R.; Johnson, J. R. J. Am. Chem. Soc. 1930, 52 (5), 2083-2090.

(14) Cairncross, A.; Roland, J. R.; Henderson, R. M.; Sheppard, W. A. J. Am. Chem. Soc., 1970, 92, 3187-3189.

(15) Cohen, T.; Schambach, R. A. J. Am. Chem. Soc. 1970, 92 (10), 3189-3190.

(16) Gooßen, L. J., Deng, G., Levy, L. M. Science 2006, 313 (5787), 662-664.

(17) Gooßen, L. J.; Linder, C.; Rodríguez, N.; Lange, P. P.; Fromm, A. Chem. Commun. 2009, 46,7173 .

(18) Lu, P.; Sanchez, C.; Cornella, J.; Larrosa, I. Org. Lett. 2009, 11 (24), 5710-5713.

(19) Hoover, J. M. Comments Inorg. Chem. 2017, 37 (4), 169-200.

(20) Nilsson, M.; Kulonen, E.; Sunner, S.; Frank, V.; Brunvoll, J.; Bunnenberg, E.; Djerassi, C.; Records, R. Acta Chem. Scand. 1966, 20, 423-426. 
(21) Guo, J.-J.; Hu, A.; Chen, Y.; Sun, J.; Tang, H.; Zuo, Z. Angew. Chem. Int. Ed. 2016, 55 (49), 15319-15322.

(22) Abderrazak, Y.; Bhattacharyya, A.; Reiser, O. Angew. Chem. Int. Ed. 2021, anie. 202100270 .

(23) Kochi, J. K. J. Am. Chem. Soc. 1962, 84 (11), 2121-2127.

(24) Shields, B. J.; Doyle, A. G. J. Am. Chem. Soc. 2016, 138 (39), 12719-12722.

(25) Treacy, S. M.; Rovis, T. J. Am. Chem. Soc. 2021, jacs.1c00687.

(26) Xia, S.; Hu, K.; Lei, C.; Jin, J. Org. Lett. 2020, 22 (4), 1385-1389.

(27) Feng, G.; Wang, X.; Jin, J. Eur. J. Org. Chem. 2019, 2019 (39), 6728-6732.

(28) Belding, L.; Chemler, S. R.; Dudding, T. J. Org. Chem. 2013, 78 (20), 10288-10297.

(29) Paderes, M. C.; Keister, J. B.; Chemler, S. R. J. Org. Chem. 2013, 78 (2), 506-515.

(30) Champagne, P. A.; Desroches, J.; Hamel, J.-D.; Vandamme, M.; Paquin, J.-F. Chem. Rev. 2015, 115 (17), 9073-9174.

(31) Yerien, D. E.; Bonesi, S.; Postigo, A. Org. Biomol. Chem. 2016, 14 (36), 8398-8427.

(32) Concurrent to this work, Ritter and coworkers described a protocol for the fluorodecarboxylation of aromatic acids that is related to a component of this study: Xu, P.; LópezRojas, P.; Ritter, T. J. Am. Chem. Soc. 2021, 143 (14), 5349-5354.

(33) Gooßen, L. J.; Thiel, W. R.; Rodríguez, N.; Linder, C.; Melzer, B. Adv. Synth. Catal. 2007, 349 (14-15), 2241-2246.

(34) Casitas, A.; Ribas, X. Chem. Sci. 2013, 4 (6), 2301.

(35) Le, C.; Chen, T. Q.; Liang, T.; Zhang, P.; MacMillan, D. W. C. Science 2018, 360 (6392), 1010-1014.

(36) Misawa, H.; Sawabe, K.; Takahara, S.; Sakuragi, H.; Tokumaru, K. Chem Lett 1988, 17 (2), 357-360. 